\title{
CIRCULARITÉ ET FONDATION TRANSCENDANTALE CHEZ LE JEUNE RICHIR. RICHIR AVEC FICHTE, KANT ET HUSSERL
}

\author{
SACHA CARLSON
}

\begin{abstract}
:
This article seeks to highlight the two founding moments of Richirian architectonics, when, in his early works, he sought a foundation for his own phenomenological approach. The first moment can be found in his work from 1979, Le rien et son apparence, which consists of an interpretation of the first version of Fichte's Doctrine of Science (1794-95). We show first that it is by reading Fichte that Richir finds a first basis for his thought; and then we show also that it is in the very depths of the text of the WL that Richir finds the question of a phenomenological imagination, which he will not cease to develop in his later works, also drawing inspiration from Kant and Husserl. The second moment is found in the Recherches phénoménologiques (1981-83), where it is the revival of the Kantian theme of transcendental illusion that allows Richir to found his approach as a paradoxical mathesis of instability.
\end{abstract}

Dans ce cadre problématique [scil. celui de la question de l'intersubjectivité en phénoménologie et chez Husserl] où, d'une certaine façon, la phénoménologie husserlienne se rejoue dans son entier, il n'est pas surprenant que la problématique de la primordialité et du Leib primordial ait été presque toujours mal comprise - cela, d'autant plus que, selon les modes intellectuelles de l'époque, il était de bon ton de "déconstruire" toute possibilité d'attestation de l'originaire, mais dans la croyance fausse ou naïve ("métaphysique"), qu'attester de l'originaire était du même coup céder à l'illusion d'une archè dont tout le reste eût dû découler. Il ne nous revient pas, ici, de montrer que c'est là une fausse lecture de la phénoménologie (et en particulier de celle de Husserl), qui confond Fundierung phénoménologique, Stiftung d'un registre architectonique sur la base d'un autre registre architectonique et Begründung métaphysique - où le registre "fondé" devrait être "dérivé" (par quelque moyen logico-ontologique) du registre "fondateur". Ainsi, était-il aussi facile qu'erroné de prétendre que le primordial husserlien et le Leib primordial étaient un "mythe" ou une "illusion" métaphysique - dans l'“esprit du temps", certes, mais sans comprendre que sur ce point celui-ci était faux de part en part ${ }^{1}$.

Marc Richir, Phantasia, imagination, affectivité (Grenoble, Jérôme Millon, Coll. Krisis, 2004). pp. 272-273. 


\section{Introduction}

Il suffit de parcourir, ne fût-ce que rapidement, les titres des différents ouvrages de Marc Richir², pour s'apercevoir que l'idée de fondement y est prégnante : il y est tantôt question de fondement (au singulier comme au pluriel ${ }^{3}$ ) ou de fondation ${ }^{4}$, tantôt de «nouvelles fondations $»^{5}$, de « refondation » ou de « refonte $»^{6}$. Or la prégnance d'un tel thème ne peut manquer d'étonner, tant il se démarque des questions caractéristiques du contexte philosophique ambiant, lorsque Richir élaborait sa propre pensée : l'époque n'était-elle pas à l'exercice du soupçon par rapport à toute instance fondationnelle, que ce soupçon s'exerçât sous la forme d'une critique logique du langage dans une perspective dite analytique, ou qu'il se déployât plutôt comme une critique de l'onto-théologie classique, le plus souvent sous la forme d'une déconstruction de la métaphysique et de son histoire? Après la mort de Dieu, n'avait-on pas célébré la mort de l'Homme lui-même, le reléguant, dans une approche se revendiquant tout à la fois de Heidegger et des sciences dites humaines, à la seule place vide d'un «signifiant zéro »?

Comment comprendre alors cette attention appuyée de Richir à la question du fondement, par où il semble remonter le courant de toute son époque ? Peut-être pourrait-on objecter que dans les expressions évoquées, la question du fondement

2 Pour une présentation globale de l'œuvre de Marc Richir en français, on peut se rapporter aux textes suivants : Richir Marc, L'écart et le rien. Conversations avec Sacha Carlson, Grenoble, Jérôme Millon, 2015 ; Schnell Alexander, Le sens se faisant. Marc Richir et la refondation de la phénoménologie transcendantale, Bruxelles, Ousia, 2011 ; Alexander Robert, Phénoménologie de l'espace-temps chez Marc Richir, Grenoble, Jérôme Millon, 2013 ; Forestier Florian, La phénoménologie génétique de Marc Richir, Springer, coll. « Phaenomenologica ", 2014 ; Carlson Sacha, "L'Essence du phénomène. La pensée de Marc Richir face à la tradition phénoménologique », in Eikasia. Revista de Filosofia $n^{\circ} 34,2010$, pp. 199-360. On pourra consulter, en espagnol, les travaux importants de Pablo Posada Varela sur et à partir de Richir. Citons entre autres : " En torno a la singladura filosófica y fenomenológica de Marc Richir ", in Eikasia 40, 2011, pp. 239-290; Pablo Posada, "Fenómeno, phantasia, affectividad. La refundición de la fenomenología en Marc Richir ", in Acta Mexicana de Fenomenología. Revista de Investigación filosófica y científica, Año 1. No. 1. Febrero de 2016, pp. 91-114; " Algunos aspectos de la fenomenología de Marc Richir », in Revista filosófica de Coimbra, no 46 (2014), pp. 397-428.

3 Au-delà du renversement copernicien (La Haye, Martinus Nijhoff, coll. Phaenomènologica $\mathrm{n}^{\circ} 73$, 1976) a pour sous-titre : "La question de la phénoménologie et de son fondement ", alors que Le rien et son apparence (Bruxelles, Ousia, 1979) a celui de "Fondements pour la phénoménologie».

4 Le premier tome des Recherches phénoménologiques (Bruxelles, Ousia, 1981) est sous-titré : «Fondation pour la phénoménologie transcendantale».

5 «Nouvelles fondations » : telle est le sous-titre de Phénoménologie en esquisses (Grenoble, Jérôme Millon, Coll. Krisis, 2000).

6 Les termes de "refondation » et de " refonte » sont couramment utilisés par Richir dans différents textes des années 2000 . 
s'entend moins comme le sol à quoi devrait ouvrir l'analyse philosophique, que comme le fondement de ou pour la phénoménologie elle-même : en ce sens, ce que vise Richir en parlant de fondement serait plutôt une certaine rigueur dans le discours et la pratique de la phénoménologie ; et une rigueur qui passe aussi par une confrontation patiente avec les auteurs et les grands systèmes de la tradition philosophique, qu'il s'agirait d'ailleurs moins de déconstruire que de traverser pour les interroger, tout en se faisant soi-même questionner par eux. Cette objection ne suffit pourtant pas à résoudre la question, si l'on s'enquiert du souci constant de Richir, dans son œuvre, d'élucider le champ de l'archaïque, de l'origine ou de l'originaire - autant de notions qui résonnent comme des harmoniques de l'idée même de fondement. Cela s'atteste de manière particulièrement frappante dans son exigence de pratiquer une phénoménologie qui soit en même temps une « philosophie transcendantale » et une " philosophie première $»^{7}$. Mais peut-on en conclure que la phénoménologie richirienne doit se comprendre comme la reprise (au sens musical du terme), fût-ce sous la forme de variations, des thèmes et motifs principaux de la tradition métaphysique ; et qu'en ce sens, l'exigence fondamentale de Richir d'un retour au champ de l'originaire soit l'indice de ce que sa pensée est en fait une transposition (toujours au sens quasi-musical du terme) possible de la quête traditionnelle du fondement, alors même que la nouvelle tonalité de la question doive nécessairement se fonder elle-même dans les structures traditionnelles de la philosophie?

On s'en doute, les choses ne sont pas si simples. Commençons par préciser qu'on aurait tort de considérer la pensée richirienne comme la répétition des structures traditionnelles de la philosophie. Ce serait se méprendre sur le sens même de ses textes, en n'y décelant pas, en particulier, l'extrême subversion qui s'y opère du cadre philosophique ancien ; et en ne voyant pas non plus la mutation profonde qui s'y produit, quoique de manière jamais thématisée, du langage et de l'écriture proprement philosophiques : écriture à même laquelle on peut sentir, en même temps qu' une accointance profonde avec les plus anciens des philosophes, le chiffre d'une étonnante nouveauté, c'est-à-dire encore de ce que je reconnais, pour ma part, comme l'indice d'une bouleversante jeunesse dans la manière de recevoir et d'approcher les questions philosophiques. C'est dire que si la question du fondement est omniprésente dans la pensée de Richir, elle n'y est jamais traitée classiquement : certes, toujours discutée avec la tradition philosophique, mais aussi à rebours de

7 Ce thème fleurit, par exemple, dans les deux tomes des Recherches phénoménologiques (Bruxelles, Ousia, 1981-83). 
cette même tradition. C'est dire, donc, aussi, qu'il ne s'agit plus de rechercher une substance censée se tenir d'elle-même, dans son autonomie ontologique, en tant qu'instance première et irréductible par rapport à quoi tout devrait être reconduit, et inversement, à partir de quoi tout le reste pût être déduit. En fait, comme je voudrais le montrer dans cet article, l'attention singulière de Richir à la question du fondement doit avant tout se comprendre comme le souci de garder le fondement comme question: la quête du fondement est en réalité une recherche de la quête elle-même, dans son inlassable mouvement vers son principe, qu' elle ne peut pourtant jamais embrasser. On comprend alors qu'il s'agit moins de retrouver un socle - commencement, principe, cause ou fondement - comme base solide qui assurerait la stabilité du questionnement philosophique, dès lors lui-même assuré de la pérennité de l'être qu'il cherche à couvrir de ses regards; il s'agit au contraire de retrouver le souffle même des questions, en rejoignant le mouvement de cette inlassable poursuite du fondement qui a animé la philosophie depuis ses origines, c'est-à-dire, à en croire Aristote, depuis son $\theta a v \mu \alpha \zeta \varepsilon \tilde{v} v$ initial. Finalement, loin de vouloir répéter le cadre ancien du questionnement, il s'agit surtout de regagner l'enfance des questions, c'est-à-dire l'enfance de l'interrogation philosophique elle-même, à laquelle doit puiser tout questionnement philosophique vivant.

Les remarques qui précèdent avaient pour but de préciser l'esprit dans lequel je me propose d'examiner ici l'architectonique richirienne. C'est qu'on ne comprendra rien à la pensée de Marc Richir si l'on ne prend pas en compte le caractère inauguralement radical et fondamental de son entreprise philosophique, par où il s'astreint à toujours reprendre les questions à la racine de leur jaillissement, c'est-àdire en s'insérant dans le courant même de la tradition philosophique, c'est-à-dire aussi en n'hésitant pas à tutoyer d'emblée, et sans scrupule aucun, les plus Grands ! Telle est l'hyperbole tout aussi bien philosophique qu' existentielle de Richir, dont il s'agit, désormais, d'essayer de mesurer si elle a été féconde, ou si elle a au contraire cédé à l'hybris qui guette sans cesse les philosophes. Dans le cadre de cette contribution, je centrerai mon propos sur la première confrontation radicale et fondatrice que je décèle dans l'œuvre de Richir. Contrairement à ce que l'on pourrait supposer, il ne s'agit pas d'une discussion serrée avec Husserl - quoique Richir ait consacré un long Mémoire de maîtrise, en 1968, à la pensée du père de la phénoménologie - ; il ne s'agit pas non plus d'un débat avec Heidegger, qui a pourtant nettement marqué les tout premiers textes de Richir - il considérait d'ailleurs son texte intitulé 
"Le rien enroulé " ${ }^{8}$, qui se déploie à partir d'une lecture de Heidegger, comme le " premier acte de naissance de sa pensée ». Je veux parler de la lecture de Fichte qu'il propose inauguralement dans sa thèse doctorale (1973). Ce texte, ensuite publié comme monographie avec pour titre: Le rien et son apparence ${ }^{9}$, constitue une lecture et une interprétation pas à pas de la Grundlage der gesamte Wissenschaftslehre («Les principes [ou : L'Assise] de la doctrine de la science») datant de 1794-1795, qui est, comme on le sait, la première version de la Wissenschaftslehre, la Doctrine de la science, à savoir l'ouvrage fondamental de Fichte. Mon hypothèse de travail est que c'est en se confrontant à ce texte que Richir a trouvé pour la première fois, si je puis dire, l'assise de sa propre pensée, d'où découlent au moins deux sillons fondamentaux de sa phénoménologie : l'un qui concerne la question de l'illusion, et qui se déploie dans un sillage kantien à travers la question de l'illusion transcendantale ; l'autre qui concerne la problématique de l'imagination, et qui finira par se développer dans un contexte husserlien.

\section{La systématique fichtéenne}

Mais il nous faut commencer par l'ancrage de la pensée richirienne dans l'œuvre fichtéenne ${ }^{10}$. On sait que la première lecture que Richir offre de Fichte, dans sa thèse doctorale de 1973 (dont la partie sur Fichte est publiée en 1979 sous le titre Le rien et son apparence), est en fait un commentaire suivi et détaillé du texte de la première Wissenschaftslehre de $1794 / 95^{11}$. Or, pour comprendre l'écart parfois presque invisible, mais systématique, que Richir creuse et maintient entre sa propre pensée et le système propre de Fichte, et par où il forge sa propre architectonique, il convient de prendre d'abord un peu de hauteur par rapport au trajet complexe qu'emprunte le texte fichtéen. Autrement dit, il convient d'abord d'en revenir à l'architectonique globale de la Wissenschaftslehre ${ }^{12}$.

8 Richir Marc, «Le Rien enroulé. Esquisse d’une pensée de la phénoménalisation », in Textures 70/7.8, Bruxelles, 1970, pp. 3-24.

9 Richir M., Le rien et son apparence, Bruxelles, Ousia, 1979.

10 J'en ai proposé une première approche dans mon article : Carlson S., «Richir avec Fichte. Remarques sur la première interprétation richirienne de la Doctrine de la science (1794/95) ", in Annales de phénoménologie $\mathrm{n}^{\circ} 16 / 2017$, pp. 63-91.

11 Fichtes Werke, hrsg. Von F. Medicus, Felix Meiner, Leipzig, 1922, Bd. I, pp. 285-317, tr. fr. d'Alexis Philonenko dans : Fichte, Euvres choisies de philosophie première, Paris, Vrin, 1964.

12 Dans ce qui suit, je m'appuie dans un premier temps sur le commentaire que Richir propose d'un texte important où, juste après la doctrine de l'Anstoss et avant la « Déduction de la représentation ", Fichte réfléchit sur le sens du chemin parcouru (Wissenschaftslehre, pp. 217-228; Fichte, Euvres choisies..., op. cit., pp. 102-108) (Richir M., Le rien..., op. cit., pp. 189-211). 
Rappelons ${ }^{13}$ que dans son texte, Fichte choisit de partir des exigences du savoir, formulées par les trois principes initiaux de la Wissenschaftslehre. C'est par là que la tâche propre de la Wissenschaftslehre peut être clairement établie : «la tâche était d'unifier les opposés, Moi et Non-Moi » ${ }^{14}$; une tâche qui prend d'abord la forme de la proposition suivante : "Le Moi se pose comme déterminé par le Non-Moi ", en laquelle on reconnaît la formule de la "représentation ", placée au sommet de la "Fondation du savoir théorique ». Il s'ensuit une analyse régressive qui caractérise la «Fondation du savoir théorique », et qui conduit le philosophe à trouver une synthèse par laquelle les opposés sont unifiés ; et dans cette synthèse, l'opposition se révèle peu à peu comme celle du fini et de l'infini, lesquels peuvent s'unifier dans l'imagination comme " pouvoir de production absolu » ${ }^{15}$ : " par l'imagination, qui unifie le contradictoire (Widersprechendes), ils peuvent être complètement unifiés ${ }^{16}$. C'est par là qu'une explication de la possibilité de la représentation, comme articulation d'un sujet et d'un objet, peut être trouvée : "le Non-Moi est lui-même un produit du Moi se déterminant soi-même, et rien d'absolu et de posé en dehors du Moi. Un Moi qui se pose comme se posant soimême, où un sujet n'est pas possible sans un objet pro-duit (hervorgebracht) de la manière qui a été décrite (la détermination du Moi, sa réflexion sur soi-même en tant qu'un (être) déterminé, n'est possible que sous la condition qu'il se limite soi-même par un opposé) ${ }^{17}$. Aussi, pour expliquer la représentation, la doctrine de l'imagination devra être complétée par la doctrine de l'Anstoss, qui seule peut expliquer l'auto-limitation du Moi susceptible d'engendrer la représentation. Dès lors, il semble clair qu'avec la doctrine de l'imagination et de l'Anstoss, la proposition placée au sommet de l'ensemble de la doctrine de la science théorique est complètement épuisée, puisque toutes les contradictions qui se trouvaient en elle ont été levées. Cependant, cela ne signifie pas pour autant que la Wissenschaftslehre elle-même soit achevée : car si la possibilité de la représentation a effectivement été expliquée, il n'a pas encore été montré comment l'articulation d'un Moi déterminant (un sujet) et d'un Non-Moi déterminé (un objet) s'engendrent concrètement

13 Pour un bref rappel des différentes interprétations classiques de la Wissenschaftslehre (Guéroult, Philonenko, etc.) par rapport à celle de Richir, que je suis ici dans l'ensemble, cf. mon article, déjà cité : « Richir avec Fichte. Remarques sur la première interprétation richirienne de la Doctrine de la science (1794/95)».

14 Wissenschaftslehre, p. 218 ; Fichte, Euvres choisies..., op. cit., p. 102 (cité et traduit par Richir in Richir M., Le rien..., op. cit., p. 190).

15 Wissenschaftslehre, p. 217 ; Fichte, Euvres choisies..., op. cit., p. 102.

16 Wissenschaftslehre, p. 218 ; Fichte, Euvres choisies..., op. cit., p. 102 (cité et traduit par Richir in Richir M., Le rien..., op. cit., p. 190).

17 Wissenschaftslehre, p. 218 ; Fichte, Euvres choisies..., op. cit., p. 102 (cité et traduit par Richir, in Richir M., Le rien..., op. cit., p. 190). 
à partir du Moi absolument posant : ce sera la tâche de la « Déduction de la représentation », dans laquelle Richir décèle une pensée implicite de la phénoménalisation, en ce que Fichte cherche à y montrer comment le double mouvement de l'activité infinie du Moi se diffracte en lui-même sous l'impact d'un choc, pour donner lieu à un double mouvement où apparaissent les deux pôles distincts que sont l'activité et la passivité, qui seront ensuite fixés et reconnus comme le sujet et l'objet d'une représentation ${ }^{18}$; cette tâche sera d'ailleurs poursuivie par la partie pratique de la Wissenschaftslehre, qui cherche à montrer que l'activité originaire du Moi sous la forme de l'imagination recèle en son fond tout aussi bien la possibilité de revenir à soi dans la pure position de soi-même, que celle de s'ouvrir à un élément hétérogène, et en ce sens, contient déjà la possibilité d'un choc sur l'activité. Autrement dit, après avoir dégagé les composantes originaires de l'esprit humain au terme de l'analyse régressive, il s'agit de reconstruire le savoir humain à partir de ses sources, en une sorte d'analyse - ou de déduction - progressive. En tout cas, comme le souligne Richir ${ }^{19}$, l'examen de la structure globale de la Wissenschaftslehre montre clairement que l'Anstoss constitue le véritable pivot de tout le texte. Celui-ci se structure en fait selon un double mouvement, dans un premier temps, de descente vers le fondement du savoir, et dans un second temps, de remontée depuis ce fondement à partir duquel se trouve déduit le savoir luimême (ainsi que le savoir du savoir), dès lors pleinement légitimé. Et le pivot qui renverse pour ainsi dire la vapeur, en faisant rebrousser l'analyse en sens inverse, n'est autre que la doctrine de l'Anstoss. C'est en ce point que se joue toute la systématique fichtéenne, et c'est donc sur cette question que va se concentrer l'approche critique de Richir.

Il faut d'abord s'interroger sur l'analyse régressive qui précède et achemine vers l'Anstoss : celle-ci consiste en une " déduction » systématique effectuée par le philosophe, à partir de la proposition problématiquement énoncée au commencement de la Doctrine de la science. C'est pourquoi un soupçon pèse sur toute la première partie du texte : car il se pourrait bien que ce qui a été déduit n'ait de réalité que dans l'esprit du philosophe, et non pas dans le Moi réel. C'est d'ailleurs ce que Fichte explique clairement lui-même : « toutes les possibilités intellectuelles (Denkmöglichkeit) que nous pensions, tout au long de notre recherche, et que nous pensions avec la conscience de les penser, étaient aussi des faits de notre conscience, en tant que nous philosophions : mais il s'agissait de faits, produits artificiellement (künstlich), selon les lois de la réflexion, par la spontanéité de notre

18 Sur la lecture richirienne de la « Déduction de la représentation », cf. « Richir avec Fichte. Remarques sur la première interprétation richirienne de la Doctrine de la science (1794/95) », art. cit.

19 Richir M., Le rien..., op. cit., pp. 198 sq. 
pouvoir de réflexion. La possibilité intellectuelle seule restante, et présentement établie, après le rejet de tout ce qui a été démontré comme faux, est tout d'abord aussi un fait artificiellement produit par la spontanéité de l'acte de philosopher : il est pour autant avéré qu'il a été élevé à la conscience (du philosophe) par la médiation de la réflexion; ou plus proprement dit : la conscience de ce fait est un fait produit par l'art $»^{20}$. Autrement dit, tout ce que l'analyse régressive obtient ne pourrait être qu'une construction philosophique à quoi ne répond rien dans la réalité $d u$ Moi. Et cela serait en contradiction avec le projet fichtéen d'une fondation du savoir : « la proposition placée au sommet de notre recherche doit être vraie ; en d'autres termes quelque chose doit lui correspondre dans notre esprit $[\ldots] »^{21}$. Et c'est pour s'en assurer que Fichte cherche à conduire son analyse vers un fait qui soit absolument indubitable. La stratégie de l'analyse de Fichte mérite d'ailleurs d'être rappelée : c'est en explorant les différentes possibilités contenues dans la proposition de départ de la Wissenschaftslehre, que l'analyse philosophique est amenée à découvrir, au cœur même de son objet (l'essence de la conscience), les traces de sa propre activité philosophante ${ }^{22}$. C'est alors que, confrontée à elle-même, la conscience philosophique découvre ce qui est nécessairement présupposé au fondement de sa propre activité ; ou, pour utiliser un langage ultérieur de Fichte, elle réalise ce qu' elle doit nécessairement faire pour pouvoir dire ce qu'elle dit - elle découvre le Tun qui fonde nécessairement son Sagen. C'est ce qui conduit la réflexion philosophique au fait originaire et indubitable de l'esprit qu'est l'Anstoss (et l'imagination), à partir d'où seulement la réflexion philosophique se trouve assurée, aux yeux de Fichte, de n'être pas « formelle » ou "vide » ${ }^{23}$ - c'est-à-dire de ne pas proférer des «affirmations vides de sens $»^{24}$. L'Anstoss est bien le fait indubitable recherché, dans la mesure où sans ce fait, rien ne serait explicable ou pensable : ni ce que le philosophe cherche à penser comme étant l'essence de la conscience, ni la pensée même du philosophe qui cherche à penser un tel fait. C'est en ce sens que l'Anstoss constitue le point de rebroussement de toute la Wissenschaftslehre: «il est clair qu'à ce point, écrit Fichte, toute la réflexion se situe à un autre degré et reçoit une autre signification $»^{25}$.

\footnotetext{
Wissenschaftslehre, pp. 219-220; OCCP, p. 103.

Wissenschaftslehre, p. 220 ; OCCP, p. 103.

... il s'agit, bien sûr, de l'activité indépendante, qui ne se réduit pas à la pensée philosophante - comme cela a parfois été soutenu -, mais, plus exactement, que l'activité du philosophe permet d'attester dans le cours de l'analyse. 


\section{Système et illusion : La critique richirienne du système fichtéen}

Reprenons donc à partir de ce renversement, que la réflexion philosophique subit à partir de l'Anstoss : car c'est à partir de là que Richir entend interroger le système fichtéen.

S'il est clair, pour Fichte, que c'est seulement à partir de là que la réflexion philosophique se trouve assurée de n'être pas vide, mais de toucher à quelque chose de réel dans l'essence de la conscience, il convient néanmoins de s'interroger : cette évidence que rencontre le philosophe peut-elle être tout simplement transférée à tout le reste de ses analyses? Autrement dit, le philosophe peut-il s'appuyer sur le fait originaire de l'Anstoss pour reconstruire ses analyses, désormais légitimées par l'évidence nouvellement acquise ? On s'en doute, c'est précisément sur ce point que porte la critique de Richir. Et il est remarquable qu'il s'en explique à l'occasion d'un examen serré d'un passage de Fichte consacré à cette question. On trouve déjà ici, en germes, l'essentiel de la critique de Fichte qui se développera ultérieurement ${ }^{26}$. Le texte de Fichte en question mérite d'être cité intégralement :

Les opposés sont dans les deux cas un subjectif et un objectif; mais ils sont en tant que tels, avant la synthèse et après elle, d'une manière très différente dans l'esprit humain. Avant la synthèse, ils sont de purs (bloss) opposés et rien de plus ; l'un est ce que l'autre n'est pas, et l'autre, ce que le premier n'est pas ; ils désignent un pur rapport (Verhältnis) et rien de plus. Ils sont quelque chose de négatif et absolument rien (schlechthin nichts) de positif [...]. Ils sont une pure pensée sans aucune réalité (Realität) ; et de plus, la pensée d'une pure relation. - Dès que l'un entre en scène (eintritt), l'autre est anéanti ; mais du fait que celui-ci ne peut entrer en scène que sous le prédicat de l'opposé (des Gegenteils) de l'autre, et que par conséquent le concept de l'autre entre en même temps en scène avec son concept et l'anéantit, celui-ci ne peut pas lui-même entrer en scène. Il n'y a donc rien de disponible (vorhanden) et il ne peut y avoir rien de disponible ; notre conscience n'est pas remplie et il n'y a en elle absolument rien de disponible. (Å vrai dire, nous n'aurions pas du tout pu entreprendre toutes nos recherches précédentes sans une illusion (Taüschung) bienfaisante de l'imagination qui, de façon non-remarquée, glissait un substrat au-dessous de ces purs opposés; nous n'aurions pas pu penser sur eux, car ils n'étaient absolument rien, et on ne peut pas réfléchir sur rien. Cette illusion, il ne fallait pas l'empêcher, et elle ne devait pas être empêchée ; son produit devait (sollte) seulement être soustrait et exclu de la somme de nos déductions, comme cela s'est produit (geschehen ist) effectivement). Après la synthèse, ils sont quelque chose (etwas) qui se laisse saisir (festhalten) dans la conscience et qui remplit

26 Notamment dans Phénoménologie et institution symbolique (Jérôme Millon, Grenoble, 1988) et La crise du sens et la phénoménologie (Jérôme Millon, Grenoble, 1990) 
également celle-ci. (Ils sont pour la réflexion, avec l'autorisation et la permission de celle-ci, ce qu' ils étaient aussi bien auparavant, mais de façon non remarquée, et avec la constante protestation de la réflexion). ${ }^{27}$

À ma connaissance - le commentaire de Richir mis à part -, ce texte tout à fait étonnant n'a pas retenu l'attention des commentateurs modernes ${ }^{28}$. Fichte y examine précisément le passage de l'analyse régressive jusqu'au fondement, à la remontée à partir du fondement, au lieu de la synthèse de l'imagination et de l'Anstoss. Or il est à première vue troublant que ce que dit Fichte à propos de ce qui précède la synthèse accentue l'impossibilité d'effectuer une telle synthèse. Avant la synthèse, explique-t-il, les opposés sont de simples pensées, c'est-à-dire « rien », et ne peuvent donc donner lieu à une synthèse réelle dans la conscience. Comment comprendre, dès lors, qu'après la synthèse, ils soient compris comme " quelque chose » qui remplisse véritablement la conscience? La solution proposée par Fichte consiste à supposer que l'analyse régressive effectuée par le philosophe sur ses propres pensées - dont rien ne garantit au départ la réalité -, se révèle finalement secrètement guidée par des « lois de la réflexion » qui garantissent la justesse du parcours philosophique par rapport à la réalité de la conscience. La réflexion est certes libre, comme Fichte l'annonçait au début de son analyse, mais cette liberté ne se déploie que selon des lois déterminées.

Dans le texte cité, Fichte s'explique sur ce point dans l'importante parenthèse, que j’ai souligné pour cette raison : la réflexion philosophique, écrit-il, est d'emblée guidée par une "illusion bienfaisante de l'imagination », qui seule permet de convertir le « rien » des pensées philosophiques, en « quelque chose » dans la conscience. En ce sens, comme le souligne Richir, tout l'édifice fichtéen semble finalement reposer sur cette « illusion bienfaisante ». Ne faut-il pas alors en interroger le statut ? Richir formule en tout cas plusieurs questions à ce sujet; et tout d'abord celle de savoir en quoi le pouvoir illusoire de l'imagination est ici bienfaisant? La réflexion n' est-elle pas mise en danger par le " pouvoir subversif de l'imagination ${ }^{29}$ ? Son caractère illusoire n'annule-t-il pas toute la Wissenschaftslehre jusqu' ici ? Autrement dit, comme le remarque explicitement Richir en faisant appel à une notion qui deviendra cardinale dans sa pensée ultérieure, Fichte ne touche-til pas ici à quelque chose comme son moment du Malin Génie $e^{30}$ : mais ce serait un moment manqué, faute d'avoir été réfléchi en tant que tel ? Il est caractéristique,

27 Wissenschaftslehre, pp. 224-225 ; Fichte, Euvres choisies..., op. cit., p. 106.

28 Il avait cependant déjà été épinglé par Hegel en 1801, dans la Differenzschrift.

29 Richir M., Le rien..., op. cit., p. 203.

30 Richir M., Le rien..., op. cit., p. 203. 
d'ailleurs, que Fichte semble sentir la difficulté, lorsque, quelques lignes plus loin, il commence son analyse de l'illusion comme suit : " à toute illusion doit (muss) s'opposer la vérité, toute illusion doit (muss) se laisser éviter ${ }^{31}$. Mais il est facile de montrer que le caractère de nécessité de cette proposition (c'est le verbe müssen qui est utilisé) s'appuie en fait sur l'opposition classique, depuis Platon, entre la vérité et l'erreur ${ }^{32}$. Et en ce sens, comme l'explique encore Richir, c'est finalement le platonisme inscrit au cœur même de la démarche fichtéenne qui autorise à résorber ce moment de subversion - celle du Malin Génie - tout juste frôlé, en affirmant que l'imagination ne peut pas produire une pure illusion, puisque dans ce cas, il n'y aurait que de l'illusion, ce qui est contradictoire, puisque qu'il n'y a d'illusion qu'au regard de la vérité. Plus loin, l'explication de Fichte s'appuie également sur un autre argument classique, celui du cogito : «Si donc maintenant il est montré, comme cela doit (soll) être montré dans le présent système, que c'est sur cette action de l'imagination que se fonde la possibilité de notre conscience de notre vie, de notre être pour nous [...], alors cette action ne peut pas être supprimée (wegfallen) [...] ; par conséquent, l'imagination n'illusionne pas (täuscht ... nicht), mais elle donne la vérité et la seule vérité possible ${ }^{33}$. Une argumentation qui, tout comme la précédente, " n'est pas absolument convaincante ${ }^{34}$, puisqu' elle ne permet pas de répondre à l'objection selon laquelle aussi bien le monde que le Moi qui se donne dans le cogito, pourraient n'être qu'une illusion cohérente, un simple produit illusoire de l'imagination ${ }^{35}$. Sur cette lancée, Richir n'hésite pas à formuler d'autres questions : si l'illusion de l'imagination est aux yeux de Fichte, «bienveillante ", en quoi celle-ci s'oppose-t-elle à cet autre type d'imagination, qui n'est que sous-entendu dans le texte, et qui serait une imagination malfaisante? Par ailleurs, on peut se demander ce qui distingue l'illusion bienfaisante de l'imagination à l'œuvre dans la première partie de la Wissenschaftslehre et la vérité censée éclater à partir de la suite (notamment dans la partie nommée la « Déduction de la représentation $»)^{36}$. Or il est remarquable que la réponse proposée par Richir, comme en passant, à cette dernière question, anticipe une thèse fondamentale développée dix ans plus tard dans les Recherches phénoménologiques. Richir explique en effet que ce qui fait la différence entre la première partie Wissenschaftslehre et la Déduction

\footnotetext{
Wissenschaftslehre, p. 227; Fichte, Euvres choisies..., op. cit., p. 108.

Cf. Richir M., Le rien..., op. cit., p. 203.

3 Wissenschaftslehre, p. 227 ; Fichte, Euvres choisies..., op. cit., p. 108, cité et traduit par Richir, in Richir M., Le rien..., op. cit., p. 202.

4 Richir M., Le rien..., op. cit., p. 202.

Cf. Richir M., Le rien..., op. cit., p. 203.

36 Cf. Carlson S., « Richir avec Fichte. Remarques sur la première interprétation richirienne de la Doctrine de la science (1794/95) », art. cit.
} 
n'est finalement qu'une « différence de niveau ${ }^{37}$ dans le déploiement de l'illusion : alors que dans un premier temps, l'illusion est censée guider la réflexion philosophique dans sa recherche de la vérité, à partir de la synthèse fondamentale de la Wissenschaftslehre, cette même illusion en vient à "prendre corps » sous la forme de la phénoménalisation. Manière de dire que la «vérité » advient non pas avec la suppression de l'illusion, mais au contraire, lorsque celle-ci advient pleinement, lorsqu'elle prend apparence, se phénoménalise. Il en ressort, curieusement, une conception du phénomène qui ne s'accorde plus avec le cadre strictement fichtéen, puisque pour Richir, le phénomène, tout comme la pensée du phénomène, doit désormais se comprendre comme indissociable de son illusion constitutive - qu'on peut alors nommer, avec Kant, illusion transcendantale. Et à y bien réfléchir, c'est ce qui fait le fonds de cette première interprétation de la Wissenschaftslehre, à savoir ce qu'il cherche à y penser comme la pure périphérie comme lieu du phénomène, et qu'il nomme aussi, à cette époque, sa distorsion originaire ${ }^{38}$, mais n'est qu'un autre nom de l'illusion transcendantale.

\section{Imagination et phénomène}

Comme on vient de le voir, l'illusion ne cesse de travailler dans les profondeurs de la systématique fichtéenne. Mais comme on l'a vu également, cette prégnance ne s'explique que parce que l'illusion s'articule étroitement à l'imagination : c'est bien l'imagination transcendantale elle-même qui trône au sommet de la Wissenschaftslehre, dans la mesure où c'est elle seulement qui permet de comprendre la synthèse originaire de la représentation, et qui rend également possible la synthèse même de l'acte philosophique ; bref, c'est à elle seule qu'il convient d'attribuer l'éminence du statut de faculté première. Or, on l'a dit, la question de l'imagination constitue l'une des lignes directrices de la pensée richirienne. Je voudrais suggérer ici que c'est en partie dans la première lecture de Fichte que s'ébauchent, pour la première fois, les linéaments de la phénoménologie richirienne de l'« imagination ». On verra alors que se trouvent en germe certaines questions axiales pour les développements plus récents de sa pensée.

Commençons par rappeler quelques traits fondamentaux de la doctrine fichtéenne de l'imagination. On se souvient que dans les textes consacrés à la synthèse de la substantialité, Fichte considère l'imagination transcendantale comme

37 Richir M., Le rien..., op. cit., p. 204.

38 Cf. en particulier son article séminal : «Phénoménalisation, distorsion, logologie. Essai sur la dernière pensée de Merleau-Ponty », in Textures 72/4.5, Bruxelles / Paris, 1972, pp. 63-114. 
la faculté qui opère l'union originaire entre le fini et l'infini, et par où la synthèse du troisième principe initialement formulé devient effectivement possible. C'est ce que Fichte confirme dans les dernières pages de la partie théorique de la Wissenschaftslehre, de manière particulièrement précise : « toute réalité (Realität), écrit Fichte, $[\ldots]$ est produite seulement par l'imagination ». Autrement dit, "c'est sur cette action de l'imagination que se fonde la possibilité de notre conscience de notre vie, de notre être pour nous, c'est-à-dire de notre être en tant que Moi $»^{39}$. Cependant, ces quelques pages, qui examinent aussi la question du système, vont encore plus loin : Fichte y explique en effet, à propos de l'économie d'ensemble de la Wissenschaftslehre dans sa partie théorique, que le philosophe se trouve finalement pris dans un conflit apparemment irréductible entre l'impossibilité (pour nous philosophe) d'effectuer la synthèse finale entre le fini et l'infini, et l'exigence (toujours pour nous), cependant, de l'effectuer. Or, l'esprit du philosophe qui, séjournant dans son conflit, réussit malgré tout cette synthèse, n' est autre que l'imagination elle-même. Par conséquent, lorsque la synthèse s'accomplit malgré tout, c'est que l'esprit du philosophe rejoint l'activité du Moi qu'il ne faisait qu'examiner du dehors jusque là, de sorte que la synthèse des opposés absolus doit non seulement se comprendre comme celle du subjectif et de l'objectif, mais aussi, en même temps, comme celle de l'esprit du philosophe et de l'activité infinie du Moi ${ }^{40}$. Mais dans ces deux cas, c'est l'imagination elle-même qui effectue la synthèse : soit qu'on la prenne à sa racine comme un flottement (schweben) ou un double mouvement originaire entre les opposés purs, dans leur jaillissement premier et co-originaire, alors qu'ils ne sont pas encore op-posés - et l'imagination se comprend alors comme la forme première de l'esprit (Gemüt) au fondement de la représentation ; soit qu'on la prenne comme la faculté par laquelle l'esprit du philosophe éprouve les opposés absolu, et l'impossibilité d'effectuer leur synthèse, alors même que c'est par cette épreuve qu'il s'élance en un mouvement qui en vient à rejoindre le mouvement originaire de l'imagination, et effectue par là, paradoxalement, la synthèse pourtant impossible à réaliser. Si l'imagination se trouve originairement au fondement du savoir comme le double mouvement qui le rend possible, le philosophe ne parvient à penser ce fondement qu'en rejoignant ce mouvement, ce qui n'est possible que s'il rencontre l'impossibilité de penser ce même fondement.

En tout cas, c'est à partir de ce texte de Fichte que Richir entend formuler deux remarques principales, qui concernent la question de l'imagination, et où se profilent déjà, significativement, certains développements ultérieurs. La première

39 Wissenschaftslehre, p. 227 ; OCCPP, p. 108 (cité et traduit par Richir in Richir M., Le rien..., op. cit., p. 202).

40 Cf. Richir M., Le rien..., op. cit., p. 204. 
remarque consiste à montrer que c'est ce caractère paradoxal de l'imagination, chez Fichte, qui permet de déceler le «trait fondamental de l'esprit fichtéen »:

Lorsque Fichte écrit que cela même (les opposés absolus) qui menace d'anéantir la possibilité d'une théorie du savoir humain, est la seule condition sous laquelle une telle théorie peut être établie. Quand il affirme que les opposés absolus, non-unifiables et complètement incommensurables au pouvoir de saisir du Moi, donc cette impossibilité de leur synthèse, sont en même temps la seule condition de possibilité de l'imagination productrice qui effectue la synthèse, il nous dit que c'est du fond même de l'impossibilité que jaillit le possible, que c'est du fond même de l'incompréhensible (de ce qui ne peut être compris, donc pris ensemble, synthétisé) que jaillit le compréhensible (ce qui peut être pris ensemble, synthétisé). La Wissenschaftslehre théorique connaît ici son point de rebroussement parce qu'elle a été "jusqu' au bout" de l'impossible, parce qu'elle a poussé jusqu'à l'opposition suprême et que c'est de l'opposition suprême que jaillit la synthèse suprême. ${ }^{41}$

D'ailleurs, comme il le précise encore, «ce "trait fondamental" ne se démentira jamais dans l'œuvre de Fichte ; au contraire, il constitue le fil conducteur permettant de relier l'une à l'autre les différentes versions de la Wissenschaftslehre; il vient même de plus en plus en avant, dès la Wissenschaftslehre de 1801. Dans une lettre à Jacobi datée du 31 mars 1804, Fichte ira jusqu'à écrire que la philosophie n'est même rien d'autre que "le comprendre de l'incompréhensible en tant que tel", où il faut penser ce comprendre comme un "comprendre médiat", c'est-à-dire un comprendre qui ne doit pas faire de l'incompréhensible quelque chose de compréhensible, mais qui doit comprendre l'incompréhensible dans son incompréhensibilité, en le laissant être comme tel ${ }^{42}$. Autrement dit, c'est ce «trait fondamental » qui permet de comprendre la continuité qu'il y a entre les différentes versions de la Wissenschaftslehre ${ }^{43}$, alors même qu' on peut aussi l'envisager comme le caractère de toute une époque : « on le découvre au cœur des innombrables "fragments philosophiques" de Novalis (1794-1799), très fortement inspirés, il est vrai, au moins au début, par la lecture de la Wissenschaftslehre 1794. Il traverse aussi de nombreux essais philosophiques de Hölderlin, notamment le très remarquable écrit intitulé La démarche de l'esprit poétique, datant des années 1798-1800 ("Période

Richir M., Le rien..., op. cit., p. 208.

42 Richir M., Le rien..., op. cit., pp. 208-209.

43 "À cet égard, précise Richir, il n’y a pas tant de différence qu'on l'a dit entre la Wissenschaftslehre de 1794 et les autres exposés de la Wissenschaftslehre à partir de 1801, puisque la Wissenschaftslehre de 1804, par exemple, se définit comme une "genèse compréhensible de l'incompréhensible" (cf. Leçon 3) et que c'est toujours ce même renversement de la Wissenschaftslehre 1974 qui sera le moteur véritable de toutes les tensions de la Wissenschaftslehre " (Richir M., Le rien..., op. cit., p. 209). 
d'Empédocle") mais encore et surtout la pensée de Hegel qui écrit, rappelons-le, dans la Préface à la Phénoménologie de l'Esprit (rédigée à la fin de 1806) : “L'esprit ne reconquiert sa vérité que dans la mesure où il se trouve soi-même dans l'absolu déchirement (in der absoluten Zerrissenheit)", et dans la Science de la logique (1812): "Quelque chose (Etwas) n'est aufgehoben que dans la mesure où il est entré (getreten ist) dans l'unité avec son opposé" - ce qui montre en passant à l'historien de la philosophie que les grands débats de l'époque, et notamment ce qu'on a appelé "l'esprit dialectique", trouve sa racine positive dans la doctrine fichtéenne de l'imagination exposée dans la Wissenschaftslehre 1794, et lui explique la convergence initiale entre le jeune romantisme allemand et la Wissenschaftslehre ${ }^{44}$. En tout cas, on pressent qu'en mettant en lumière ce " trait fondamental » centré sur la question de l'imagination, c'est sa filiation spirituelle que le jeune Richir entend également sonder ${ }^{45}$, c'est-à-dire aussi bien le «trait fondamental» de sa propre pensée. Et de fait, le caractère de redoublement propre à l'imagination ne se rapproche-t-il pas de ce que le jeune phénoménologue cherchait à penser, dans ses premiers travaux, comme la logologie qui caractérise le mouvement même de la pensée à l'état naissant, et que la pensée philosophique se doit d'épouser au plus près dans son propre mouvement ${ }^{46}$ ? Et n'est-il pas caractéristique, à cet égard, que si c'est chez Max Loreau que Richir a d'abord puisé la notion même de logologie ${ }^{47}$, ce soit finalement chez un contemporain de Fichte - Novalis - qu'il en retrouvera le premier usage philosophique?

La deuxième remarque de Richir concerne, parallèlement à la première, le statut précis à conférer à l'imagination. Car en effet, si c'est bien la question de l'imagination qui constitue le fil conducteur de l'œuvre fichtéenne, et marque en même temps le caractère de toute son époque, il convient d'en situer précisément le concept. Il faut donc lever certains « malentendus». Un certain nombre d'équivoques se trouvent d'ailleurs déjà dans le texte même de la Wissenschaftslehre, comme cela a été aperçu précédemment, et qu’on peut résumer comme suit : une première indécision tient à ce qu'on ne comprend pas très bien, chez Fichte, si l'imagination désigne une faculté transcendantale comme source du savoir, enfouie dans le système inconscient de l'esprit humain, ou bien si elle désigne un

44 Richir M., Le rien..., op. cit., p. 209.

45 Le livre publié sous le titre Au-delà du renversement copernicien (op. cit.) comporte clairement un chapitre intitulé : « Notre insertion historique positive : la rupture de l'Idéalisme Allemand (titre du chapitre IX).

46 Cf. Richir M., "La Défenestration ", L'ARC n 46 : Merleau-Ponty, Aix en Provence, 1971, pp. 31-42 ; ainsi que "Phénoménalisation, distorsion, logologie. Essai sur la dernière pensée de Merleau-Ponty ", art. cit.

47 Cf. par exemple : «Pour une cosmologie de l'Hourloupe », Critique n² 298, Paris, 1971, pp. 228-253. 
acte conscient de l'esprit - bref, s'il s'agit d'une activité productrice des schèmes transcendantaux structurant les actes de la première série du Moi, ou bien s'il s'agit d'une activité (re-)productrice d'images, relevant de la conscience naturelle, et pouvant éventuellement être réeffectuée par le philosophe, comme acte relevant de la seconde série du Moi. Corrélativement, une deuxième indécision joue dans le texte fichtéen : c'est qu'on ne comprend pas bien non plus si l'imagination caractérise en propre la production illusoire d'erreurs, de mensonges ou de simulacres, ou bien si elle concourt à prendre en vue la réalité - bref, si l'imagination est simplement la faculté de la «fantaisie », la «folle du logis », ou bien si elle permet d'accéder à la vérité ? Et s'il y a une illusion de l'imagination, comme l'établit clairement le texte de Fichte, toute la question est bien de savoir, comme Richir le demandait, dans quelle mesure cette imagination peut être convertie sans reste en « illusion bienfaisante ». Quoi qu'il en soit, à cette première série de malentendus s'en accompagne une deuxième, qui caractérise la réception de la Wissenschaftslehre par ses contemporains. Richir met ici en évidence une mauvaise interprétation, qui peut expliquer, paradoxalement, l'immense écho que connut la Wissenschaftslehre dans les milieux littéraires allemands de la fin du XVIIIe siècle : c'est que « l'Einbildungskraft [scil. chez Fichte] n'est pas la Phantasie dont se réclameront de plus en plus les romantiques, en se séparant graduellement de Fichte ${ }^{48}$. Par ailleurs : " celui qui chercha sans doute à fonder une doctrine de la création artistique sur la doctrine fichtéenne de l'Einbildungskraft, et ce, de la manière la plus conséquente et la plus rigoureuse, fut sans doute Hölderlin qui n’a jamais appartenu à l'“école romantique" regroupée autour de l'Athenäum $»^{49}$.

Il est en tout cas caractéristique que ces questions sur le statut ambivalent de l'imagination, que Richir pose comme en passant au texte fichtéen, rejailliront plus tard dans son œuvre, comme si elles n'avaient cessé d'y travailler souterrainement. Pour le montrer, on pourrait déjà évoquer les Recherches phénoménologiques (1981-8350), où le phénomène est pris dans le mouvement même de son illusion transcendantale constitutive, c' est-à-dire en prenant en compte l'influence le plus souvent inaperçue, comme dit Kant, de l'imagination sur la Raison théorique - je vais y venir. Mais je voudrais surtout évoquer un passage particulièrement instructif des Méditations phénoménologiques ${ }^{51}$, où Richir rapproche le sens commun phénoménologique de l'Einfühlung (intro-pathie) et corrélativement,

\footnotetext{
Richir M., Le rien..., op. cit., p. 210.

Ibid.

Richir M., Recherches phénoménologiques, 2 tomes, Bruxelles, Ousia, pp. 1981-83.

51 Richir M., Méditations phénoménologiques. Phénoménologie et phénoménologie du langage, Jérôme Millon, coll. Krisis, Grenoble, 1992.
} 
d'une conception renouvelée de l'imagination. Il écrit en effet : "L'Einfühlung est en réalité indissociable de l'imagination, non pas du tout au sens de "projection" d'image ou d'images, mais au sens de ressources cachées et/ou implicites, en tout cas indéfinies, constituant le fonds commun de l'expérience de “chacun” "52. C'est pourquoi, «il faut corrélativement penser une transformation très profonde du statut de l'imagination : la pluralité phénoménologique des mondes relève, en un sens, de l'imagination, c'est que la distinction ne peut plus être strictement faite, dans le champ phénoménologique, entre le "réel" et l' "imaginé", parce que cette distinction relève déjà de l'institution symbolique $»^{53}$. Autrement dit : " loin de devoir être asservie à un "réel" dont elle serait vouée à n'être que la variation ou la reproduction, elle en fait partie intégrante, en tant qu' elle en est, elle aussi, constitutive. Loin d'être presqu'absurdement une quelconque "faculté des images", reproduisant plus ou moins bien, selon ses règles et ses dérèglements, le réel in absentia - et en fait, rien de détruit plus définitivement l'imagination que l'image - elle est plus fondamentalement pouvoir de formation, et pouvoir schématisant (Kant), Einbildungskraft qui ne procède pas par image, ni selon l'arbitraire ou la liberté de sa "fantaisie", mais selon les échanges et compositions baroques " ${ }^{54}$. On l'aura compris, c'est l'ensemble des conceptions traditionnelles de l'imagination que Richir entend révoquer dans ce court texte : l'imagination n'y est plus comprise comme une reproduction (spéculaire) du réel ${ }^{55}$, ou comme la représentation d'une chose absente : c'est que l'imagination que cherche à penser Richir est en fait une imagination sans images. Il s'agit en fait d'une faculté formatrice et productrice, qui n'est pas soumis au joug d'un modèle préexistant (chose réelle ou concept), mais qui se déploie librement, et à laquelle la conscience vigile peut s'adonner, bien qu' elle n'en soit jamais le maître : l'imagination pénètre jusqu'à l'intimité de notre regard, si bien qu'on ne peut pas décider quelle est la part du réel et la part de l'imaginé, qui s'entrelacent constamment pour former le tissu de l'expérience. C'est pourquoi aussi, il n'est plus possible de procéder à la distinction classique entre l'image externe (l'image-copie, comme le tableau) et l'image interne (la « représentation » ou l' « image mentale»). Finalement, l'imagination que Richir cherche ici à décrire n'est autre que la faculté qui schématise, qui génère la dynamique propre au transcendantal, c'est-à-dire le rythme même du mouvement de la phénoménalisation. En ce sens, l'analyse phénoménologique de l'imagination semble devoir

\footnotetext{
Richir M., Méditations..., op. cit., p. 194.

Richir M., Méditations..., op. cit., p. 194.

Richir M., Méditations..., op. cit., pp. 194-195.

Dès lors, comme le précise Richir, « l'imagination n'a rien avoir avec l'imaginaire lacanien, c'est-àdire avec le spéculaire » (Richir M., Méditations..., op. cit., p.196).
} 
se mouvoir dans le cadre critique kantien, et il faudrait montrer plus précisément que c'est moins la Critique de la raison pure que la Critique de la faculté de juger qui inspire ici Richir ${ }^{56}$.

Or, le schématisme est-il le dernier mot de l'imagination phénoménologiquement réduite ? Il faudrait pouvoir montrer également comment la reprise originale de Richir sur la question se fraie un autre chemin, parallèle, à partir de Phénoménologie en esquisses (2000) ${ }^{57}$. À partir de cet ouvrage, en effet, Richir poursuit sur la même lancée, en cherchant à dissocier l' "imagination " phénoménologique de l'image ; mais cette fois-ci, c'est en s'appuyant sur les analyses de Husserl, et en particuliers sur les analyses publiées dans le volume XXIII des Husserliana, que Richir fait sa propre avancée ${ }^{58}$. Il ne peut être question de déployer ici toute la problématique, dont on sait qu'elle donnera à la pensée richirienne un nouveau départ. Je me bornerai donc à rappeler l'essentiel ${ }^{59}$, et tout d'abord que la distinction qui devient désormais axiale, n'est plus tant celle entre l'image interne (ou mentale) et l'image avec un support physique externe, ni même celle entre le réel et l'imaginaire, mais celle entre l'imagination (Einbildung, Imagination) et la phantasia (Phantasie $)^{60}$, tous les deux devant clairement se distinguer du schématisme. Dans ce contexte, l'imagination - qui peut avoir ou non un support physique externe - se définit comme un acte intentionnel où s'entremêlent indissociablement deux intentionnalités : celle qui vise la chose imaginée, que Husserl nomme le Bildsujet (par exemple le Panthéon, qui est représenté sur le tableau que je regarde), et celle qui vise l'image elle-même, que Husserl nomme le Bildobjekt (par exemple le tableau lui-même, non pas en tant que simple chose sensible, mais en tant qu'il représente autre chose que lui-même). Cette analyse permet donc de rendre compte précisément du statut phénoménologique de l'image : celle-ci,

56 Je m'y emploie dans des travaux en cours de publication.

57 Richir M., Phénoménologie en esquisses. Nouvelles fondations, Grenoble, Jérôme Millon, 2000.

58 Husserl Edmund, Phantasie, Bildebewusstsein, Erinnerung, édité par E. Marbach, coll. Husserliana $\mathrm{n}^{\circ}$ XXIII, Dordrecht-Boston-London, Kluwer Academics Publishers, 1980. Traduction française par R. Kassis et J.F. Pestureau (revue par M. Richir : Phantasia, conscience d'image, souvenir, Grenoble, Éditions J. Millon, Grenoble, 2002.

59 Je m'inspire ici largement des explications de Richir dans son texte : « Imagination et Phantasia chez Husserl ", in J. Benoist et V. Gérard (éds.), Lectures de Husserl, Paris, Ellipses, 2010, pp. 143-158. Je étudié cette question dans mon article : "Phantasia et imagination : perspectives phénoménologiques (Husserl, Sartre, Richir) », Eikasia (www.revistadefilosofia.com) n66, septembre 2015, pp. 19-58.

60 Le terme allemand Phantasie, dans ce contexte, n'a en effet pas d'équivalent satisfaisant en français. Il pourrait certes être rendu par "fantaisie » ou "fantasme ", mais on sait que ces deux termes désignent aujourd'hui tout autre chose. Aussi, dans Phénoménologie en esquisses, Richir proposera inauguralement de traduire par le grec phantasia. Il sera suivi en cela non seulement par les traducteurs de Hua XXIII, mais aussi, semble-t-il, par la communauté husserlienne francophone. 
qu'elle soit interne ou externe, n'est rien de real (ce n'est pas une chose mondaine, transcendante par rapport à la conscience); mais elle n'est pas non plus quelque chose de réellement (reell) vécu dans l'immanence de la conscience. Autrement dit, il ne s'agit pas de quelque chose qui puisse tout simplement être pris en vue pour les besoins de l'analyse. Dans les termes de Husserl, on dira qu' elle n'a qu'un statut intentionnel ${ }^{61}$, et c'est pourquoi son analyse phénoménologique ne pourra pas être à proprement parler une analyse de l'image, qui en tant que telle n'existe pas, mais bien plutôt une analyse de l'intentionnalité spécifique à la conscience d'image. Or, précisément, la difficulté d'une telle analyse est que cet unique acte intentionnel d'imaginer relève en réalité d'une intentionnalité double, où l'appréhension (Auffassung) de l'image est indissociable de l'appréhension de l'objet mis en image ${ }^{62}$, ces deux intentionnalités étant, comme l'écrit Husserl, irrémédiablement " enchevêtrées l'une dans l'autre "63. C'est aussi ce qui explique qu'il n'y a pas d'intentionnalité qui vise proprement le Bildobjekt, puisqu'en elle-même (abstraction faite de l'intentionnalité qui vise le Bildsujet), elle reste "non effectuée » ou « inaccomplie ${ }^{64}$; ce qui signifie aussi que le Bildobjekt n'est jamais posé, mais n'est que la médiation d'une position (celle du Bildsujet), qui n'est en fait, dès lors, qu'une quasi-position. C'est d'ailleurs aussi la raison pour laquelle le fait d'examiner les détails d'un objet ne fait pas partie essentiellement de l'intentionnalité imaginative. $\mathrm{Si}$, pour reprendre l'exemple célèbre de Alain, lorsque je regarde une carte postale du Panthéon, je peux revenir sur celle-ci pour compter les colonnes de l'église, ce n'est pas sur l'image elle-même (le Bildobjekt) que je reviens, mais c'est le Bildsujet que je vise, avec l'intention de regarder tel ou tel détail. Mais lorsque le support physique fait défaut, il m'est impossible de revenir sur le même Bildsujet avec une autre intention; ne reste alors que l'image, qui se volatilise lorsque j'essaie de la poser pour l'examiner comme quelque chose de réel.

Or, de l'imagination - avec ou sans support externe -, il convient de distinguer la phantasia ${ }^{65}$. Par ce terme, Husserl désigne l'acte par lequel $j$ ' "imagine »-en allemand : "ich phantasiere " - toutes sortes de choses, d'être, de situations ou de paysages, qui ne sont pas forcément supposés pouvoir se rencontrer dans la réalité : ainsi, « les petits anges » ou les « centaures ». D'où viennent ces figures, qui ne

61 Cf. Phénoménologie en esquisses (PE), Grenoble, Jérôme Millon, 2000, p. 63.

62 Cf. PE, pp. 62 sqq.

63 Hua XXIII, p. 27.

64 Cf. Hua XIII, texte $\mathrm{n}^{\circ} 16$.

65 ... distinction introduite dans le cours de 1904/05 (texte ${ }^{\circ} 1$ de Hua XXIII), même si le vocabulaire de Husserl est un peu flottant, et si la distinction est sans doute moins franche chez Husserl que ce qu'en retire Richir : il y aurait tout un travail à faire pour situer l'écart entre les textes husserliens et la reprise richirienne de la problématique. 
peuvent manifestement pas être des imitations de la réalité, ou même des recompositions de fragments de choses existantes. La thèse de Husserl consiste à dire que ces « choses" proviennent de la phantasia, et que le lieu de leur déploiement n'est pas le monde réel, mais un tout autre monde que Husserl nomme le Phantasiewelt. La phantasia doit donc se distinguer fondamentalement de l'imagination, puisqu'ici, il ne s'agit plus de viser un Bildsujet supposé pouvoir appartenir au monde réel - bien que ce ne soit pas toujours effectivement le cas -, même si c'est à travers la médiation d'un Bildobjekt; mais il s'agit au contraire de viser quelque chose de tout à fait différent : lorsque nous "phantasions", "le monde effectivement réel sombre presque sous nos regards, mais en nous faisant tout de même sentir un peu son être-là, de sorte qu'une légère conscience de l'apparence colore en permanence les formations-de-phantasia ${ }^{66}$. Autrement dit, avec la phantasia, nous ne trouvons plus la double intentionnalité caractéristique de la conscience d'image ; bien au contraire, comme l'explique Richir, « la phantasia, et les phantasiai, ne sont pas, tout au moins intrinsèquement [...], des images, mais directement, des apparitions $"{ }^{67}$. Et ces apparitions se caractérisent ${ }^{68}$ par leur aspect protéiforme ou protéique (proteusartig), par la discontinuité de leur surgissement " en éclair » (blitzhalt) dans le cours censé être continu du temps, et par leur intermittence dans ce même supposé continuum temporel ; et corrélativement, par le fait que ce qui y apparaît est non présent. Si les apparitions de phantasia se distinguent d'emblée de la conscience d'image en ce qu'il s'y agit précisément d'apparitions, et non pas de présentifications, ce sont ces caractères, qui sont propres aux apparitions de phantasia, qui permettent de distinguer celles-ci des apparitions perceptives, c'est-à-dire, finalement, de la simple hallucination. Richir commente la situation comme suit : " par le premier caractère, il faut entendre que, alors que c'est le même objet qui est aperçu par la conscience, ses apparitions changent sans cesse, et ce, de manière discontinue, par décrochages, par exemple aussi bien de formes que de couleurs, comme quelque chose d'ombreux, de fuyant et fluctuant, sans donc que les apparitions s'enchaînent les unes aux autres de manière cohérente comme dans le cas de la perception. Par surcroît, s'il y a en elles de la couleur, ce n'est pas au même sens que la couleur perçue : c'est comme une sorte de gris qui, sans être perceptif, est comme un "vide indicible"» (Hua XXIII, 59). L'apparition de phantasia est donc déjà, en ce sens, insaisissable comme telle. 2) Le caractère de discontinuité de son surgissement signifie à son tour qu'elle jaillit en un éclair (aufblitzen) sans arriver à se stabiliser ou à se fixer : elle est comme l'Einfall qui

66 Hua XXIII, p. 42.

67 PE, p. 68.

68 Cf. $\$ \$ 28-29$ du cours de 1904/05, Hua XXIII, pp. 58-65. 
advient inopinément, vient subitement à l'esprit. 3) Selon le troisième caractère, l'apparition de phantasia peut disparaître complètement aussi vite qu' elle a surgi, mais, dans sa fugacité même elle peut tout aussi bien revenir, resurgir pour disparaître à nouveau, éventuellement sous une forme tellement métamorphosée (caractère protéiforme) que nous pouvons tout d'abord croire apercevoir un autre "objet". Enfin, 4) si l'on comprend mieux par ces trois premiers caractères que "ce qui" y apparaît (les guillemets signifiant que l'on ne peut jamais distinguer ce "ce qui" par une quiddité) est non présent, le paradoxe extrême est que les apparitions de phantasia elles-mêmes n'ont aucun rapport au présent (Hua XXIII, 79) »69. On sera alors en droit de se demander ce que peut être, concrètement, une apparition non présente d'un non présent : il ne s'agit même pas d'une ombre, mais de l'ombre fuyante d'une ombre elle-même depuis toujours disparue. Ce sont ces questions qui conduiront d'ailleurs Richir à distinguer progressivement entre, d'une part, les "phantasiai pures", qui n'apparaissent en effet pas plus à la conscience vigile que des " "ombres" (mais ombres de rien), des "fantômes" (mais fantômes d'aucun être) ou des "feux follets" tout à fait immatériels » ${ }^{70}$, et qui ne peuvent donc être attestées qu'indirectement par l'analyse phénoménologique ; et d'autre part, les "phantasiai perceptives", qui désignent ce type particulier de phantasiai en lesquelles il y a "perception" (Perzeption) de quelque chose qui est au-delà, aussi bien du réel perçu en Wahrnehmung, que du fictif comme objet intentionnel de l'imagination ${ }^{71}:$ l'exemple type est celui du personnage de théâtre "incarné " par le comédien ; dans ce cas, je " perçois " (perzipiere) bien en phantasia le personnage, mais au-delà de la perception (Wahrnehmung) du corps et des paroles du comédien, bien que ce soit à partir de ce corps perçu que je peux "phantasier » le personnage, alors même qu'il ne s'agit pas non plus d'un Bildobjekt figurant un Bildsujet : il n'y a pas ici d'imagination, car ce qui est perçu, loin d'être un objet imaginé, reste proprement infigurable, comme l'est le personnage de théâtre ou de roman, dont on sent bien qu'il n'est jamais réductible à sa mise en image, laquelle paraît toujours forcément « infidèle $»^{72}$.

\footnotetext{
"Imagination et phantasia chez Husserl », art. cit., p. 8.

Fragments phénoménologiques sur le langage, op. cit., p. 4.

Cf. ibid., p. 8.

2 La notion de phantasia perceptive est introduite par Husserl dans le texte $n^{\circ} 18$ de Hua XXIII, précisément à propos du théâtre, et sera reprise et élargie par Richir, jusqu'à devenir centrale dans sa pensée, puisque les phantasiai perceptives seront identifiées aux phénomènes de langage, alors même que les phantasiai pures seront associées aux phénomènes hors langage. Pour s'introduire à cette question tout à fait capitale, on lira en particulier les Fragments phénoménologiques sur le langage (Grenoble, Jérôme Millon, 2008).
} 


\section{Phénoménologie et illusion transcendantales}

Après avoir suivi le premier sillon à partir de Fichte qui poursuit la question de l'imagination jusqu'aux développements à partir de Husserl dans les années 2000, revenons à l'autre rameau que nous évoquions, qui concerne la question de l'illusion dans le cadre d'une philosophie première ou transcendantale. Car la phénoménologie de Richir ne s'est jamais contentée de se déployer comme une seule phénoménologie de l'imaginaire - fût-il " phantastique »-, mais elle s'est aussi d'emblée conçue comme une phénoménologie transcendantale. On a vu que l'œuvre de Fichte constitue sur ce point un moment inaugural dans la démarche de Richir, lorsqu'il choisit d'emprunter la voie tracée par son illustre prédécesseur pour se frayer un chemin vers la question du fondement - fût-ce pour en critiquer, chemin faisant, le principe. Pourtant, c'est surtout l'œuvre de Kant qui a permis à Richir de poursuivre cette problématique rencontrée au cœur de la Wissenschaftslehre : la question de l'illusion transcendantale constitue en ce sens comme un pivot qui a permis de Richir de remonter de Fichte à Kant. Cela se vérifie de manière exemplaire dans l'ouvrage que Richir considérait lui-même comme le « deuxième acte de naissance » de sa pensée : les deux tomes des Recherches phénoménologiques ${ }^{73}$, sur lesquelles je voudrais m'arrêter à présent pour montrer comment la question d'une philosophie transcendantale s'y articule rigoureusement à la question de l'illusion transcendantale, en proposant en quelque sorte une solution à l'aporie féconde rencontrée inauguralement chez Fichte. Je le montrerai à m'arrêtant successivement sur trois « moments " marquant de l'analyse richirienne : la question de la philosophie transcendantale, la problématique de l'illusion transcendantale, et la notion de réflexion transcendantale.

\section{L'idée d'une philosophie transcendantale}

Commençons par l'idée d'une philosophie transcendantale, en rappelant que chez Kant lui-même, de qui Richir s'inspire ici, cette idée ne se laisse pas définir simplement ${ }^{74}$. On connait bien sûr la définition canonique du terme «transcendantal » dans la Critique de la raison pure ${ }^{75}:$ «j'appelle transcendantale, écrit Kant,

Marc Richir, Recherches phénoménologiques, 2 tomes, Bruxelles, Ousia, 1981-1983.

74 Ce qu'a excellemment mis en évidence Frank Pierobon dans son ouvrage remarquable, duquel je m’inspire ici : Kant et la fondation architectonique de la métaphysique, coll. " Krisis ", Éditions Jérôme Millon, Grenoble, 1990.

75 Kant Immanuel, Kritik der reinen Vernunft (Éditions Reclam, Stuttgart, 1995). Je cite le texte en me référant selon l'usage, à la première édition (1781) par la lettre A, et à la seconde édition (1787) par la lettre B. Je cite la traduction d'Alexandre J.L. Delarnarre et François Marty, dans l'édition 
toute connaissance qui s'occupe en général non pas tant d'objet que de notre mode de connaissance des objets en tant qu'il est possible en général ${ }^{76}$. Est donc dit transcendantal ce qui peut être connu (a priori) de notre mode de connaissance ( $a$ priori). Et dans la mesure où la philosophie se définit comme le système des connaissances purement rationnelles, la philosophie transcendantale doit donc désigner le système des connaissances transcendantales, c'est-dire la connaissance pure du système de l'esprit humain ${ }^{77}$ : nous sommes donc apparemment au plus proche de Fichte. Pourtant, face à l'éminence d'une telle visée, et dans l'attente d'une telle exposition systématique, le lecteur de Kant ne peut être que déçu, lorsque l'auteur de la Critique semble se dérober : " cette philosophie est encore trop à son tour pour commencer ». Il assure certes que " ce système est possible » et "même qu'il n' est pas tellement vaste qu'on ne puisse en espérer l'achèvement complet ${ }^{78}$, mais c'est pour conclure que «la philosophie est l'idée d'une simple science possible, qui n'est donnée nulle part in concreto $»^{79}$. C'est pourquoi, le point de départ, plus modeste, ne peut être qu'une propédeutique, sous la forme d'une critique de la raison, qui vise à clarifier non pas les connaissances philosophiques elles-mêmes, mais les sources du pouvoir de connaitre en général, en établissant par là même les limites de la connaissance purement rationnelle, tout en en prévenant les erreurs et les déviations possibles. En ce sens, on comprend aussi combien est ambigüe et complexe la situation de la critique par rapport à la philosophie transcendantale comme telle : en tant qu' elle examine les sources de toute connaissance, et donc aussi les concepts-souches de la raison pure, la critique est forcément amenée à esquisser le plan de la philosophie transcendantale, alors même qu'elle ne peut pas le réaliser par elle-même. C'est que si la philosophie transcendantale est censée se déployer uniquement dans le champ de la raison pure, purement a priori, la critique doit forcément trouver son point de départ dans la connaissance déjà existant - qu'elle soit vraie ou illusoire. La critique, et la philosophie transcendantale telle qu'elle s'esquisse à même la critique, souffre de cette étrange posture de viser les conditions de possibilité a priori de la connaissance à partir de ce qui est donné a posteriori ${ }^{80}$.

Qu'en est-il alors de la philosophie transcendantale que vise Richir, et dont on a dit qu'elle s'inspirait de Kant ? Les remarques qui précèdent prennent tout

\footnotetext{
complète des Euvres philosophiques d'Emmanuel Kant, parue aux Éditions Gallimard, 1980, Bibliothèque de la Pléïade, sous la direction de F. Alquié.

76 Kant Immanuel, Kritik der..., op. cit., A12 / B25.

77 Cf. ibid. ainsi que, par exemple, A841 / B869.

78 Kant Immanuel, Kritik der..., op. cit., A12 / B26.

79 Kant Immanuel, Kritik der..., op. cit., A838 / B66.

80 Sur tout ceci, voir Pierobon F., Kant et la fondation architectonique de la métaphysique, op. cit.
} 
leur sens quand on réalise que Richir aborde le criticisme kantien à partir d'une difficulté parallèle, lorsque sous le terme de philosophie transcendantale, il cherche sonder l'esprit global du kantisme :

[...] la philosophie transcendantale kantienne est toujours recherche des conditions de possibilité a priori d'un fait (la connaissance humaine, la moralité, le sentiment esthétique, l'impression d'une finalité naturelle) qui est cependant mis en suspens dans la recherche : si un tel fait est possible, alors il faut que... En outre, selon l'esprit du renversement copernicien, comme un tel fait est toujours un fait humain, et comme les conditions de possibilité a priori ne peuvent être recherchées qu'en l'esprit humain, la recherche de ces conditions de possibilité est en quelque sorte immanente à l'esprit humain, ou plus précisément au fait modalisé par le comme si : il s'agit toujours de rechercher dans le "creux" du fait ses propres conditions de possibilité a priori, et cela n'est effectivement possible que si le fait n'est plus considéré comme fait brut et positif, mais comme fait existant sur le mode du comme si - comme si ce fait pouvait aussi bien être possible qu'impossible, par où s'ouvre le champ de ses conditions de possibilité, mais ce champ, également possible a priori, ne s'avère réel que dans la mesure où le fait est aussi fait réel. ${ }^{81}$

La difficulté relevée par Richir est donc bien analogue à celle que nous venons de relever, puisqu'elle vient aussi du fait que la recherche des conditions de possibilité a priori s'amorce toujours dans un fait $a$ posteriori qu'il s'agit de clarifier. Bref, la difficulté tient en ce que l'a priori est toujours envisagé comme l'a priori d'un a posteriori toujours déjà donné. En ce sens, comme le souligne Richir, la démarche critique et transcendantale se meut forcément dans un "cercle " 82 : si l'a priori est pensé dans la Critique comme ce qui fonde l'a posteriori, il faut dire de même, en toute rigueur critique, que l'a posteriori fonde lui aussi, d'une certaine manière, l'a priori, puisque c'est seulement depuis l'a posteriori que «s'indique " l'a priori. Il y a bien circularité, puisque si l'a posteriori renvoie toujours à son a priori, celui-ci ramène inexorablement à celui-là.

Il est en tout cas caractéristique que c'est à partir de l'analyse de cette circularité décelée au cœur de la démarche kantienne, que Richir en vient à proposer sa propre conception de la philosophie transcendantale. L'idée que Richir poursuit inlassablement à travers tous les chemins sinueux de ses Recherches est que la démarche de fondation transcendantale est intrinsèquement liée à cette circularité : il ne faut donc pas chercher vainement à l'éliminer, mais bien plutôt à la creuser,

81 Richir M., Recherches..., Tome 1, op. cit., p. 69.

82 Cf. Richir M., Recherches..., Tome 1, op. cit., p. 33. 
à la développer et à la faire prendre apparence - pour tout dire, à la phénoménaliser. Et cette idée méthodologique, qui semble guider l'écriture richirienne dès les premières pages de son texte, offre aussi, d'entrée de jeu, l'idée même de la philosophie transcendantale telle que la vise Richir. Car dans ce contexte renouvelé, la démarche transcendantale comme recherche du fondement ne peut plus se concevoir à la manière de Kant, comme la prise en vue a priori de l'a priori; car on l'a vu, l'a priori pur, censé surgir de lui-même et se donner dans le pur a priori, ne peut être qu'une fiction modalisée par un comme si. Bien plus, il s'avère que si l'a priori se donne toujours a posteriori, il se constitue comme l'illusion nécessaire d'un a priori : l'a priori tel qu'il se donne supposément comme le fondement absolument certain, se tenant de lui-même et portant en soi les conditions de sa propre validité est en fait une illusion transcendantale. Par là, on peut déjà comprendre que si la philosophie transcendantale proprement richirienne se déploie à partir de la circularité relevée chez Kant, c'est qu'elle se déploie explicitement sur le lieu même d'une illusion transcendantale. Et l'on comprend également que si cette philosophie vise encore à mettre au jour un fondement, celui-ci devra être paradoxalement conçu comme traversé de part en part par l'illusion transcendantale, c'est-à-dire aussi, par l'erreur, le rêve, l'hallucination, ou la folie.

\section{L'illusion transcendantale}

Cette première approche de la problématique nous a déjà permis de constater que la démarche richirienne n'est pas à proprement parler kantienne. Cependant, il est tout à fait caractéristique que si l'analyse s'engage à partir d'une critique de la philosophie transcendantale kantienne, cette critique est précisément rendue possible par la conceptualité kantienne elle-même, et en particulier par la notion d'illusion transcendantale : tel est en effet le deuxième moment marquant de l'analyse richirienne. Sur cette question, je commencerai par remarquer que chez Kant aussi bien que chez Richir, le terme d' « illusion " peut avoir deux significations voisines. En termes richiriens, il peut tout d'abord désigner l'a priori lui-même, comme ce qui est visé par la pensée comme l'être en lui-même - le fondement -, mais dont l'analyse critique montre qu'il n'est qu'une illusion, c'est-à-dire un simulacre ou un mirage qui n'a pas l'être que la pensée lui prête. Mais le terme peut aussi signifier le processus même de constitution de l'illusion : j'entends par là le mouvement de la pensée, a posteriori, par lequel un a priori est posé comme se tenant de soi a priori. L'illusion transcendantale désigne alors le processus en vertu duquel la pensée projette des horizons qui lui permettent de "fonctionner ", alors même que ces horizons prennent nécessairement l'apparence d'un être qui 
se tient de soi, indépendamment de la pensée qui les pense - et cette apparence elle-même relève alors d'une illusion au premier sens. Bien sûr, ces deux significations s'entremêlent constamment dans les textes, dans la mesure où l'une désigne un processus et l'autre le résultat de ce même processus. Prenons un exemple : comme l'explique Kant dans la Dialectique transcendantale, la connaissance de la nature requiert de prendre le monde comme un tout. Or, de ce tout, précise-t-il, on ne peut rien connaître : dans les termes de Kant, il s'agit d'une Idée régulatrice, c'est-à-dire d'un horizon que la connaissance projette elle-même pour assurer son propre déploiement, mais qui peut toujours se muer en illusion transcendantale dès lors qu'on cherche à en connaître le statut ontologique (le monde est-il fini ou infini, a-t-il un commencement dans le temps ou bien n'en a-t-il pas, etc. ? - il s'agit, bien sûr, des antinomies). Ici, l'illusion est plutôt à prendre au premier sens, comme l'apparence illusoire d'un monde connu dans sa totalité, alors même que c'est en critiquant le processus illusoire (second sens de l'illusion) que Kant en viendra à résoudre l'antinomie envisagée.

Cela étant, peut-on dire que Richir reprend tout simplement la conception kantienne de l'illusion transcendantale ? À première vue, cela semble être le cas : car c'est en écho presque direct à Kant que Richir déploie sa propre problématique de l'illusion. Il cherche en effet à établir que la pensée ne peut pas penser sans s'imaginer qu'il y a toujours de la pensée avant elle. Plus précisément, il montre que la pensée laissée à son libre cours, devient spontanément imagination, et pose cet «avant » comme l’a priori de son propre déploiement, qui est censé lui donné son assise tout en l'ancrant simultanément dans l'être et dans le concept. Il s'agit bien, ici aussi, d'une illusion transcendantale, et de même structure que l'illusion kantienne, dans la mesure où il s'agit également d'un processus illusoire par lequel la pensée projette une apparence apparaissant illusoirement comme indépendante de la pensée qui la projette. Cependant, il convient de préciser que la conception richirienne de l'illusion transcendantale n'est pas non plus strictement kantienne. C'est que cette conception relève chez Richir de deux présupposés critiques incompatibles avec le cadre kantien. Premièrement, il présuppose que l'ensemble de l'exercice naturel de la pensée est inséparable de son illusion transcendantale, en vertu de laquelle la pensée s'apparaît toujours comme assurée de son propre déroulement - comme se sachant toujours déjà avant et après elle-même, c'est-àdire comme se précédant et se succédant systématiquement elle-même. Autrement dit, si chez Kant l'illusion transcendantale menaçait exclusivement le champ de la raison pure, en tant qu'il se trouve à l'écart de la sensibilité, pour Richir, c'est tout le champ de la pensée et de la connaissance qui se voit traversé par l'illusion. Mais il y a plus : deuxièmement, Richir présuppose aussi que l'illusion transcendantale 
reste intacte au sein même de la pensée transcendantale : impossible, donc, de s'installer comme "spectateur impartial » pour prendre en vue l'exercice naturel de la pensée entraîné par son illusion constitutive. Bien au contraire, le philosophe transcendantal est lui-même emporté par le mouvement de son illusion propre; et c'est d'ailleurs ce qui explique la circularité caractéristique de la démarche transcendantale qui a déjà été relevée. Il n'est donc plus possible de supposer, comme chez Kant, que la réflexion transcendantale puisse se déployer sereinement, comme depuis la hauteur d'un tribunal souverain de la Raison, à l'écart de toute ruse et de toute tromperie.

On comprendra alors que ce changement de cadre apporte toute une série de questions et de difficultés inédites. La première d'entre celles-ci consiste assurément à interroger la possibilité même d'analyser l'illusion transcendantale comme telle : comment décrire cette illusion qu'on ne peut éviter ${ }^{83}$ et qui traverse l'ensemble de la pensée, sans même épargner la pensée du philosophe qui cherche à la penser? Bref, comment approcher l'illusion sans s'illusionner? Dans son texte, Richir ne thématise pas véritablement sa méthodologie d'approche, qui se déploie cependant progressivement au fil de ses analyses inlassablement répétées et creusées, selon une logique de progression circulaire dont on ne comprend sans doute pas immédiatement la pertinence. Peut-être est-ce parce que seule la ruse est à même de déjouer les pièges de l'illusion ? Et en ce sens, peut-être est-ce plutôt à travers l'écriture tout à fait singulière des Recherches qu'on aura le plus de chance de saisir la structure et le style propre de cette pensée ? Je me contenterai d'avancer ici que l'enjeu, pour Richir, est de ne pas être totalement obnubilé par l'illusion transcendantale; mais ceci n'est possible que si le philosophe conserve une certaine distance par rapport à l'apparence illusoire que produit sa propre pensée ; et si donc l'analyse parvient à apercevoir, en même temps que cette apparence, le mouvement même de la pensée qui la projette. C'est pourquoi la pensée transcendantale n'aura d'autre solution que d'accorder son propre rythme avec celui du mouvement de l'illusion, mais sans toutefois y adhérer complètement. Autrement dit, c'est par son écriture non linéaire, et pour ainsi dire en spirales, que Richir cherche à donner apparence au mouvement même de l'illusion, par où il cherche en même temps à y échapper.

83 Kant lui-même explique bien que ce type d'illusion ne résulte pas d'un défaut d'attention à la règle logique. Il précise d'ailleurs : «c'est là une illusion qu'on ne saurait éviter, pas plus que nous ne pourrions éviter que la mer ne nous paraisse plus élevée au large qu'auprès du rivage » (Kant Immanuel, Kritik der..., op. cit., A297/B353) ; car « nous avons affaire à une illusion naturelle et inévitable, qui repose elle-même sur des principes subjectifs et les donne pour des principes objectifs [...] » (Kant Immanuel, Kritik der..., op. cit., A298/B354). 


\section{La réflexion transcendantale}

On en vient alors au troisième moment que je propose de relever, et qui concerne le mouvement ou le rythme même de la réflexion, en tant qu' elle doit bien s'encorder, d'une certaine manière, à l'illusion. Cependant, il faut, ici aussi, s'entendre sur ce que désigne le terme même de réflexion, qui est loin d'être univoque, même si l'on en reste, par choix méthodologique, aux textes kantiens. On sait que le terme, issu de l'optique, ne désigne plus chez Kant la projection spéculaire et l'appréhension empirique de sa propre image, mais bien plutôt la prise en vue de la pensée par elle-même, par où seulement le sujet peut se rendre présent à lui-même. Mais il convient alors de situer plus précisément cet acte. On peut le comprendre tout d'abord de manière élémentaire, comme l'opération coextensive de tout jugement et de toute connaissance, en tant qu' elle instaure la conscience de l'unité de la synthèse dans son concept ${ }^{84}: c^{\prime}$ est ainsi que Kant envisage parfois la réflexion comme l'un des moments à l'origine des concepts empiriques, où les différences entre des représentations perceptives sont réfléchies dans leurs unité85; et parfois il désigne aussi la réflexion logique comme l'acte qui consiste à comparer des concepts entre eux ${ }^{86}$. Cependant, de ce premier type de réflexions, qu'on pourrait qualifier de "naturelles »- quoiqu' elles puissent être a priori -, Kant distingue encore ce qu'il nomme la réflexion transcendantale, laquelle s'emploie plutôt à rattacher chaque représentation à son « lieu transcendantal », pour déterminer de quelle faculté il est l'objet ${ }^{87}$. Autrement dit, la réflexion transcendantale,

84 C'est cette visée générale du sens de la réflexion qu'on trouve dans l'Anthropologie d'un point de vue pragmatique, où Kant écrit : "L'expérience est une connaissance empirique, mais la connaissance (puisqu'elle repose sur des jugements) requiert la réflexion (reflexio), par conséquent la conscience de l'activité qui compose la diversité de la représentation selon la règle de son unité, c'est-dire le concept et la pensée en général (différente de l’intuition)» (\$37).

Ainsi, dans les cours de Logique publiés par Jäsche, Kant explique que « les actes logiques de l'entendement qui produisent les concepts selon leur forme sont : la comparaison [...] ; la réflexion [Reflexion] c'est-dire la prise en considération [Ueberlegung] de la manière dont diverses représentations peuvent être saisies [begriffen] dans une conscience ; enfin l'abstraction [...] ». Et il précise : "Pour faire des concepts à partir des représentations des sensations, il faut donc comparer, réfléchir et abstraire, car ces trois opérations sont les conditions générales et essentielles de production de tout concept en général - Par exemple je vois un pin, un saule et un tilleul. En comparant tout d'abord ces objets entre eux, je remarque qu'ils diffèrent les uns des autres du point de vue du tronc, des branches, des feuilles, etc. ; mais si ensuite je réfléchis uniquement à ce qu'ils ont de commun entre eux, le tronc, les branches et les feuilles-mêmes et si je fais abstraction de leur taille, de leur configuration, etc. j'obtiens un concept d'arbre» (Ak, IX, pp. 94-95, tr. Guillermit, p. 103). eux dans l'entendement, pour savoir si deux concepts contiennent la même chose, s'ils se contredisent ou non [...] » (Kant Immanuel, Kritik der..., op. cit., A279/B335). 
pour Kant, est un acte qui relève spécifiquement de l'analyse philosophique, et si elle est qualifiée de transcendantale, c'est surtout pour marquer son appartenance à la philosophie transcendantale.

Ce bref rappel était nécessaire, dans la mesure où si Richir reprend explicitement la notion kantienne de réflexion transcendantale, il en modifie sensiblement le sens : il ne s'agit plus à proprement parler, chez lui, de ce qui caractérise en propre la réflexion du philosophe censée porter son regard impartialement sur les effectuations de la conscience. Mais il ne s'agit plus non plus d'une simple réflexion naturelle. Richir évoque le plus souvent la réflexion transcendantale comme une réflexion proprement phénoménologique, c'est-à-dire comme celle de l'apparence elle-même - comme une " phénoménalisation-réflexion " ${ }^{88}$, comme la réflexion à même laquelle se phénoménalise l'apparence, non pas purement a priori, ni a posteriori, mais précisément entre les deux -, de laquelle il distingue alors une " réflexion seconde ${ }^{89}$, qui provient de "notre réflexion ${ }^{90}$ philosophante, et qui abstrait des structures reconnaissables et analysables de l'apparence. Ainsi, Richir pourra parfois distinguer la " réflexion abstractive » (ou l' "abstraction transcendantale $»^{91}$ ) de la réflexion proprement transcendantale ${ }^{92}$. Mais cette distinction apparait moins nette, dès lors qu'on s'aperçoit qu'il s'agit finalement dans les deux cas de la même réflexion, mais qui est prise en vue selon deux angles différents : la réflexion proprement transcendantale désigne la réflexion intrinsèque de l'apparence, que Richir nomme parfois aussi la " réflexivité » du phénomène, et la réflexion abstractive désigne la réflexion notre réflexion, lorsque nous cherchons à penser la première réflexion. Finalement, on retrouve ici une autre forme de la circularité de laquelle nous étions parti : la réflexivité interne au phénomène n'est pensable qu'à partir de l'abstraction philosophique, alors que celle-ci n'est possible qu'à partir de celle-là. Mais cette impossibilité de discriminer totalement

88 Cf. Richir M., Recherches..., Tome 2, op. cit., p. 123.

89 Cf. Richir M., Recherches..., Tome 2, op. cit., p. 124.

90 Cf. Richir M., Recherches..., Tome 2, op. cit., p. 133.

91 Cf. Richir M., Recherches..., Tome 2, op. cit., pp. 136 \& 138.

92 Voir par exemple les textes suivants : "C'est notre réflexion elle-même, notre pensée, qui introduit ces deux pôles, ou qui les déplie en une sorte de structure transcendantale du phénomène, qui n'est rien d'autre, pour ainsi dire, que l'effet transcendantal de la réflexion que nous sommes en train d'accomplir du phénomène " (Richir M., Recherches..., Tome 2, op. cit., p. 133). "L'abstraction transcendantale que nous effectuons par notre réflexion, comme la figure unique, abstraite par nous, de l'illusion transcendantale, clignotant entre l'universalité d'une diffusion infinie du centre et la singularité de sa fixation arbitraire » (Richir M., Recherches..., Tome 2, op. cit., p. 135). « Nous divisons par notre réflexion pour la facilité de l'exposition, étant entendu, donc, que cette division n'en est qu' un effet, et un effet déformant en ce qu'il a de structurant pour l'illusion transcendantale, par où cette structure de division doit autant à notre réflexion qu'à ce qui doit se jouer dans la réflexion même du phénomène en l'illusion de sa fixité » (Richir M., Recherches..., Tome 2, op. cit., p. 150). 
la réflexion du phénomène et la réflexion philosophique se comprend, lorsqu'on se souvient que l'illusion transcendantale pénètre jusqu'au cœur de la philosophie transcendantale elle-même. On comprend ainsi que l'illusion transcendantale se trouve à la base aussi bien du mouvement de la conscience, que du mouvement de la réflexion philosophique. Et c'est donc à partir de l'illusion qu'il convient d'approcher ce mouvement réflexif. Comme l'écrit Richir, il y a :

[...] une réflexion a posteriori dans laquelle se constitue l'a priori comme a priori qui n'apparaît jamais comme tel qu'a posteriori ; par cette réflexion s'engendre l'illusion nécessaire qu'il y a un a priori, et que l'a posteriori coïncide avec l'a priori; par là même, cette illusion est aussi l'illusion d'une réflexivité réciproque de l'a priori et de l'a posteriori, c'est-à-dire, en fait, l'illusion d'une subjectivité transcendantale en laquelle s'établirait la coïncidence, l'adéquation ou la transparence de l'un à l'autre - cela même que Husserl baptise évidence. ${ }^{93}$

Autrement dit, le mouvement de l'illusion transcendantale correspond à celui d'une réflexion secrètement logée dans la pensée, qui tend à faire coïncider l'a posteriori depuis où elle se déploie et l'a priori qu' elle projette. De plus, le lieu que la réflexion projette comme son lieu propre où son mouvement aboutirait par la coïncidence de l'a priori et de l'a posteriori, prend la forme d'un subjectivité transcendantale : on mesure alors, en passant, que la discussion entreprise par Richir concerne non seulement le criticisme kantien, mais aussi bien la phénoménologie transcendantale husserlienne : si le père de la phénoménologie envisageait la démarche transcendantale comme le retour réflexif d'un subjectivité transcendantale sur elle-même, Richir s'emploie ici aussi à montrer qu'une telle subjectivité prise comme pur a priori supposé se présenter dans sa pureté à même la réflexion du cogito, ne relève en fait que d'une illusion transcendantale, dont il s'agit précisément de déplier le mouvement.

Cela se précise si l'on examine cet autre concept utilisé par Richir pour rendre compte du mouvement propre à l'illusion transcendantale, celui de rétrojection:

Le pur a priori, l'a priori en tant que tel, est insaisissable en soi, mais il est seulement, pour ainsi dire désigné comme tel depuis l'a posteriori, dans la rétrojection transcendantale du phénomène : telle est l'instance critique transcendantale, en un sens quasi ou hyperkantien, de la phénoménologie transcendantale, qu'en celle-ci s'indique un

93 Richir M., Recherches..., Tome 1, op. cit., p. 44. 
pur a priori insaisissable comme tel, sinon justement par là médiation nécessaire de l'illusion transcendantale. ${ }^{94}$

On comprend donc que si l'a priori n'apparaît jamais qu'a posteriori, il est cependant toujours " rétrojeté » a posteriori dans l'a priori, raison pour laquelle il apparaît précisément comme un a priori. Et c'est ce mouvement de "rétrojection ", toujours enfoui dans la pensée qui cherche à se penser, qu'il s'agit de déplier. Plus concrètement, comme le montrent les textes, cette rétrojection prend le visage de ce que Richir appelle encore une "précession " : c'est par là que l'a priori semble toujours précéder l'a posteriori, alors même que l'a priori n'apparaît jamais qu'a posteriori; et c'est précisément cette "semblance » du caractère apriorique de ce qui n'est pourtant jamais qu'a posteriori que Richir désigne comme une précession. Rétrojection et précession doivent donc être pensées ensemble, en ce qu' elles constituent le rythme même de la réflexion ou de l'illusion transcendantale qu'on cherche à analyser : car comme l'écrit Richir, cette réflexion n'est finalement rien d'autre qu'une " rétrojection a posteriori dans l'a priori, ou la rétrocession transcendantale d'une précession transcendantale toujours déjà manquée ${ }^{95}$. Si la rétrojection désigne le mouvement en tant qu'il s'effectue depuis l'a posteriori, la précession en désigne l'accomplissement par où l'a priori paraît toujours déjà précéder l'a posteriori. Le mouvement de l'illusion semble donc emporter la réflexion dans une course indéfinie, où la pensée court comme après son ombre.

\section{En guise de conclusion (provisoire)}

Il nous faut admettre qu'au terme de notre parcours, l'analyse que nous avons suivie semble plutôt avoir suscité la perplexité. On a certes montré que le double axe, pour le phénoménologie richirienne, de l'imagination et l'illusion transcendantale, ont été découverts et engagés dans la lecture inaugurale de Fichte. Mais en quoi, peut-on se demander à présent, ces deux axes constituent-ils une assise pour la phénoménologie richirienne, comme le titre de cet article l'annonçait ? Les développements dont nous avons cherché à rendre compte, semblent plutôt nous plonger dans l'embarras. D'une part, en suivant les analyses richiriennes de

94 Richir M., Recherches..., Tome 1, op. cit., pp. 148-149.

95 Richir M., Recherches..., Tome 1, op. cit., pp. 167-168. 
l'imagination - qui s'ancrent dans sa lecture de la Wissenschaftslehre fichtéenne, rencontrent la problématique kantienne du schématisme transcendantal et finissent par se déployer à partir de la problématique husserlienne de la phantasia - on a surtout relevé le caractère insaisissable des apparitions de phantasia, qui loin de se déployer dans le monde des objets bien découpés de la perception, n'apparaissent pas véritablement, mais transparaissent néanmoins à l'écart de toute présentation (Gegenwärtigung) ou présentification (Vergegenwärtigung), comme des ombres vacillant entre leur apparition et leur disparition, de manière discontinue, par intermittence et comme " en éclair " (selon les caractères relevés par Husserl). Comment donc cette dimension «phantastique » de l'expérience peut-elle apparaître pour phénoménologue censé en rendre compte? D’autre part, en accompagnant la visée richirienne d'une philosophie transcendantale - qui s'ancre, elle aussi, dans la lecture inaugurale de Fichte et se déploie ensuite dans l' " hyperkantisme " poursuivi dans les Recherches phénoménologiques -, nous en sommes venu à nous interroger : la réflexion qui poursuit le cercle infini de l'a prio$r i$ et de l'a posteriori ne se perd-elle pas inexorablement dans une course infernale sans but ni fin ? Et ce qui s'ouvre par là à la pensée est-il autre chose que le pur chaos de son propre égarement ? La réflexion n'y trouve-t-elle pas rien d'autre que les soubresauts de son propre épuisement? Bref, y a-t-il véritablement élucidation philosophique du champ de l'originaire, conformément à l'idée même d'une philosophie transcendantale ? Et corrélativement, quelque chose peut-il apparaître à partir de ce mouvement apparemment anarchique - et peut-on y articuler la possibilité d'une phénoménologie, conformément au projet formulé par Richir?

C'est une question qu'au terme de cette contribution, je choisi de réserver pour un travail à paraître prochainement, où je montre que ce caractère paradoxal de la phénoménologie richirienne qui consiste à vouloir penser des phénomènes foncièrement imperceptibles, et donc en un sens « inapparents ", ne vient pas d'une quelconque idéologie pseudo-gnostique qui favoriserait ce qui se dérobe - ce qui est voilé ou caché -, mais vient plutôt de l'exigence de penser le concret de l'expérience - en quoi consiste finalement le phénomène concret - à partir de son mouvement intrinsèque. C'est donc en cherchant à rendre visible le mouvement du phénomène que le phénoménologue déploie son exercice, non pas tant comme un exercice du voir ou de la vision de ce qui se montre à lui, mais comme un exercice $d u$ regard en mouvement, qui jamais ne se fixe sur ce qui est apparu, mais cherche au contraire à épouser la geste de l'apparence dans son inlassable parution. Ainsi, on comprendra que le mouvement même du phénomène en sa phénoménalisation n'est ni celui, centripète, qui conduirait à rassembler le vécu autour d'un centre originaire censé engloutir à terme l'expérience dans un archi-phénomène (Vie, 
Dieu ou Ego : peu importe ici); ni celui, centrifuge, qui conduirait l'expérience à se disséminer irrémédiablement. Il s'agit d'un double mouvement dont la forme élémentaire est celle du clignotement, que Richir avait initialement découvert chez Fichte, lorsque celui-ci parle du flottement (schweben) de l'imagination : un clignotement, explique Richir, qui n'est autre que le double mouvement des apparences entre leur apparition et leur disparition, entre leur « enroulement » comme « rassemblement d'une diversité in-finie miroitant comme la phénoménalité in-finie du phénomène " et leur déroulement ou leur dispersion de la même diversité, c'est-à-dire aussi entre leur concentration ou leur fixation, entre leur dissémination ou excentration ${ }^{96}$. Et comme il me faudra le montrer également, ce double mouvement ne peut apparaître concrètement à la pensée du philosophe qui cherche à en rendre compte, que si celle-ci en épouse partiellement le rythme, en se déployant donc comme double mouvement de la réflexion philosophique. Ce double mouvement n'est pourtant pas celui, chaotique, qui se disperse dans une multitude de fragments sans ordre ni cohérence - quoi que les fragments ou variations sont une technique que Richir a utilisée dès le milieu des années 2000. On montrera alors que c'est encore une fois la dialectique fichtéenne - mais cette fois-ci profondément transformée, par la prise en compte de la question du sublime tel que Kant l'aborde dans la troisième Critique - qui constitue la matrice philosophique du rythme - c'est-à-dire de l'ordre de son mouvement : kinèseos taxis, disait Platon (Lois, 665 a), -, propre à la pensée phénoménologique-transcendantale de Marc Richir, tel qu'on peut la lire d'un bout à l'autre de son œuvre.

Sacha Carlson, docteur en philosophie, est actuellement chercheur à la Faculté des sciences humaines de l'Université Charles (Prague). Il est également musicien compositeur.

96 Cf. Richir M., Phénomène, temps et êtres, Grenoble, Jérôme Millon, 1987, pp. 26-27. 